\title{
FEROS Abundance Analysis of 21 Bulgelike SMR Stars
}

\author{
Marina Trevisan $^{1} \dagger$, Beatriz Barbuy ${ }^{1}$, M. Grenon ${ }^{2}$, B. Gustafsson ${ }^{3}$ \\ and L. Pompéia ${ }^{4}$ \\ ${ }^{1}$ Universidade de São Paulo, IAG, Rua do Matão 1226, São Paulo 05508-900, Brazil \\ ${ }^{2}$ Observatoire de Genève; Chemin des Maillettes 51, CH-1290 Sauverny, Switzerland \\ ${ }^{3}$ Dept. of Astronomy \& Space Physics, Uppsala University, SE-75120 Uppsala, Sweden \\ ${ }^{4}$ Universidade do Vale do Paraíba, São José dos Campos 12244-000, Brazil
}

\begin{abstract}
We analyze a sample of 21 super-metal-rich (SMR) stars, using high-resolution échelle spectra obtained with the Fiber-fed Extended Range Optical Spectrograph at the $1.5 \mathrm{~m}$ ESO telescope. The metallicities are in the range $0.07 \leqslant[\mathrm{Fe} / \mathrm{H}] \leqslant 0.45,3$ of them in common with Pompéia et al. (2009). Geneva photometry, astrometric data from Hipparcos, and radial velocities from CORAVEL are available for these stars. The peculiar kinematics suggests the thin disk close to the bulge as the probable birthplace of these stars (Grenon 1999). From Hipparcos data, it appears that the turnoff of this population indicates an age of 10-11 Gyr (Grenon 1999). Detailed analysis of the sample stars is carried out, and atmospheric parameters are derived from spectroscopic and photometric determinations. Oxygen abundances of these stars are derived, and $[0 / \mathrm{Fe}]$ overabundances up to +0.35 are found.
\end{abstract}

Keywords. stars: abundances, fundamental parameters, atmospheres, late-type - Galaxy: solar neighbourhood

\section{Introduction}

Grenon $(1989,1999)$ selected a sample of about 6000 dwarf stars from the NLTT catalogue (see Grenon 1999), and studied them by means of Geneva photometry, radial velocity and Hipparcos astrometry. A sub-sample of SMR stars was revealed. In this work we report preliminary oxygen abundances for 21 of these SMR stars. Our aim is to shed light on the origin of this population and to place it in the global scenario of the Galaxy.

\section{Observations, Stellar Parameters and Oxygen Abundance}

Optical spectra were obtained using the FEROS spectrograph (Kaufer et al. 2000) at the $1.52 \mathrm{~m}$ telescope at ESO, La Silla. The total wavelength coverage is 3560-9200 $\AA$ with a resolving power $(\mathrm{R}=\lambda / \Delta \lambda)$ of 48,000 . The data reduction was carried out through a pipeline package for reductions of FEROS data, in MIDAS environment.

The surface gravities $\log g$ were derived using Hipparcos parallaxes $\pi$. The spectroscopic parameters $\left[\mathrm{T}_{\text {eff }}, \mathrm{v}_{\mathrm{t}}\right]$ were obtained by fixing trigonometric surface gravities and imposing excitation equilibrium for $\mathrm{Fe} I$ lines and ionization equilibrium for $\mathrm{Fe} I$ and $\mathrm{Fe}$ II. Independence between equivalent widths and the abundances of $\mathrm{Fe} I$ lines was imposed to determine $\mathrm{v}_{\mathrm{t}}$.

Oxygen abundances were derived from the [OI] $6300.3 \AA$ line for 16 stars in the sample, by fitting the synthetic to the observed spectra. The spectrum synthesis code is

$\dagger$ The present work has been supported by FAPESP fellowship \#08/50198-3. 


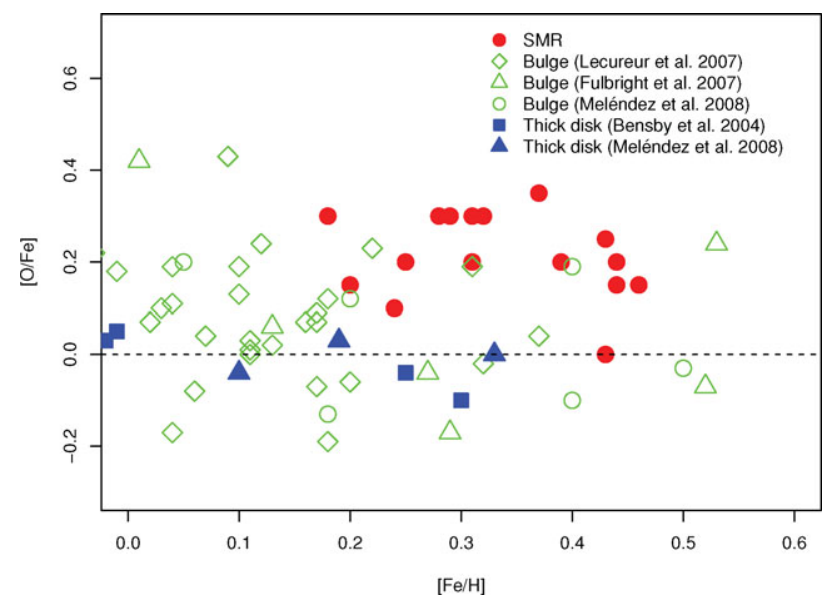

Figure 1. Derived [0/Fe] values compared with data for thick disk (Bensby et al. 2004) and bulge (Lecureur et al. 2007, Fulbright et al. 2007, Melèndez et al. 2008).

described in Cayrel et al. (1991) and Barbuy et al. (2003). Photospheric 1D models for the sample were extracted from the MARCS grid (Gustafsson et al. 2008).

\section{Conclusion}

Stellar parameters of 21 SMR stars were derived from high resolution spectra obtained with FEROS. Oxygen abundance were obtained for 16 of these stars. Values in the range $+0.10<[0 / \mathrm{Fe}]<+0.35$ were found, except for HD 77338, which has a solar 0/Fe ratio. In Figure 1 we compare our results with those for the galactic bulge and thick disk. The oxygen overabundance $(<[0 / \mathrm{Fe}]>\approx 0.2)$ suggests that these stars are bulge-like, probably formed in the inner disk at early times.

\section{References}

Barbuy, B., Perrin, M.-N., Katz, D., Coelho, P., Cayrel, R., Spite, M., \& van’t Veer-Menneret, C. $2003, A \mathscr{E} A, 404,661$

Bensby, T., Feltzing, S., \& Lundström, I. 2004, A\&A, 415, 155.

Cayrel, R., Perrin, M.-N., Barbuy, B., \& Buser, R. 1991, A\&A, 247, 108

Grenon, M. 1989, Ap\&SS, 156, 29

Grenon, M. 1999, Ap\&SS, 265, 331

Grevesse, N. \& Sauval, J. N. 1998, SSRev, 85, 161

Gustafsson, B., Edvardsson, B., Eriksson, K., Jørgensen, U. G., Nordlund, Å., \& Plez, B. 2008, $A \mathscr{G} A, 486,951$

Fulbright, J. P., McWilliam, A., Rich, \& R. Michael. 2007, ApJ, 661, 1152

Kaufer, A., Stahl, O., Tubbesing, S., Norregaard, P., Avila, G., Francois, P., Pasquini, L., \& Pizzella, A. 2000, Proc. SPIE, 4008, 459

Lecureur, A., Hill, V., Zoccali, M., Barbuy, B., Gómez, A., Minniti, D., Ortolani, S., \& Renzini, A. $2007, A \mathscr{E} A, 465,799$

Melèndez, J., Asplund, M., Alves-Brito, A., Cunha, K., Barbuy, B., Bessell, M. S., Chiappini, C., Freeman, K. C., Ramírez, I., Smith, V. V., \& Yong, D. 2008, A\& $A$ A, 484, 21

Pompéia et al. 2009, in preparation.

Stetson, P. B. \& Pancino, E. 2008, PASP, 120, 1332S 